Invited review

\title{
Structure, mechanism and therapeutic utility of immunosuppressive oligonucleotides
}

\author{
Defne Bayik $^{\mathrm{a}, \mathrm{b}}$, Ihsan Gursel ${ }^{\mathrm{b}, * *}$, Dennis M. Klinman ${ }^{\mathrm{a}, *}$ \\ a Cancer and Inflammation Program, Frederick National Laboratory for Cancer Research, Frederick, MD 21702, USA \\ ${ }^{\mathrm{b}}$ Bilkent University, Molecular Biology and Genetic Department, Therapeutic ODN Research Laboratory, Ankara, Turkey
}

\section{A R T I C L E I N F O}

\section{Article history:}

Received 13 November 2015

Accepted 13 November 2015

Available online 15 January 2016

\section{Keywords:}

CpG oligonucleotide

IRF8

IRF5

Dendritic cell

TLR9

\begin{abstract}
A B S T R A C T
Synthetic oligodeoxynucleotides that can down-regulate cellular elements of the immune system have been developed and are being widely studied in preclinical models. These agents vary in sequence, mechanism of action, and cellular target(s) but share the ability to suppress a plethora of inflammatory responses. This work reviews the types of immunosuppressive oligodeoxynucleotide (Sup ODN) and compares their therapeutic activity against diseases characterized by pathologic levels of immune stimulation ranging from autoimmunity to septic shock to cancer (see graphical abstract). The mechanism(s) underlying the efficacy of Sup ODN and the influence size, sequence and nucleotide backbone on function are considered.
\end{abstract}

Published by Elsevier Ltd.

\section{Contents}

1. Introduction

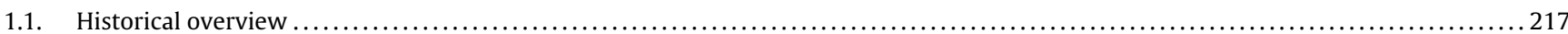

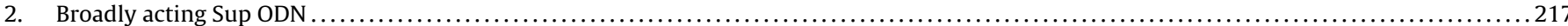

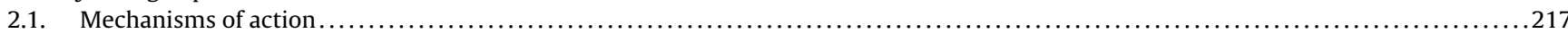

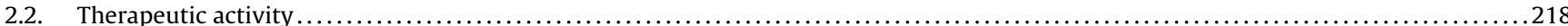

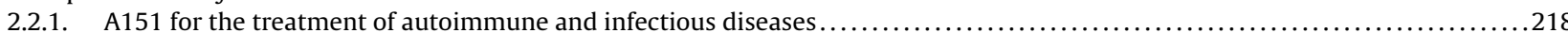

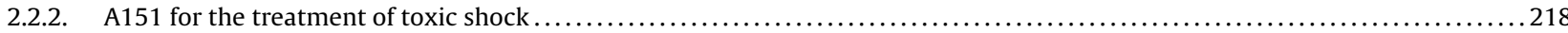

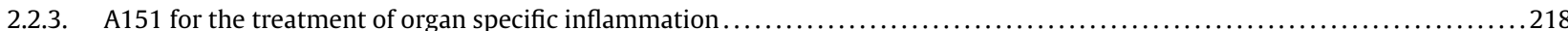

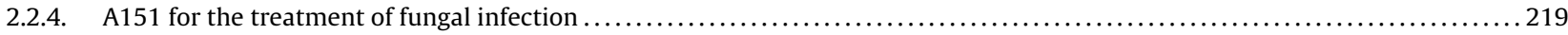

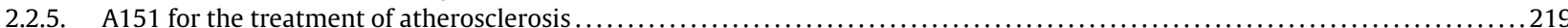

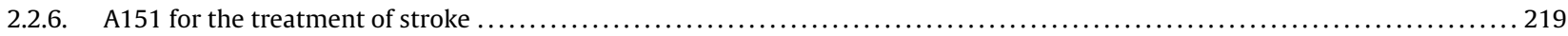

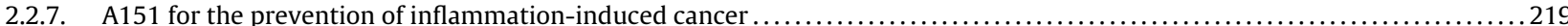

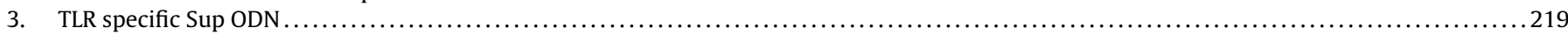

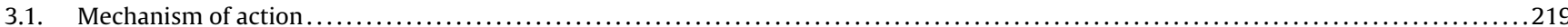

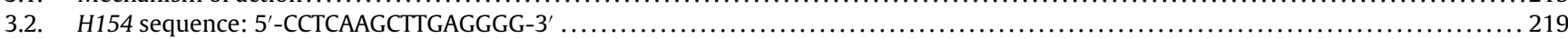

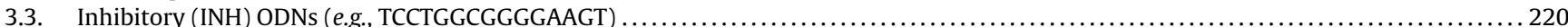

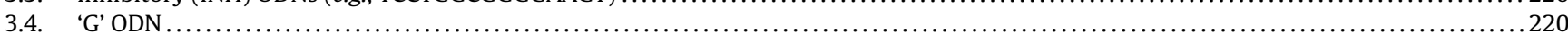

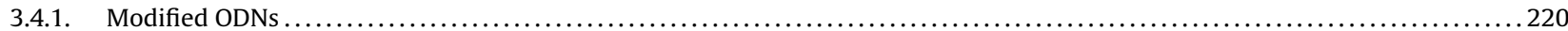

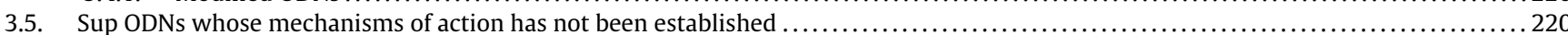

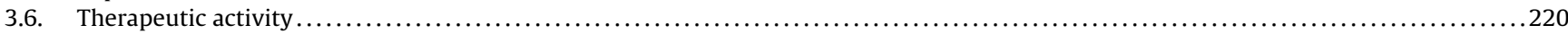

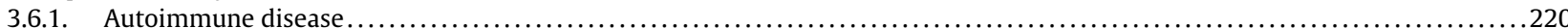

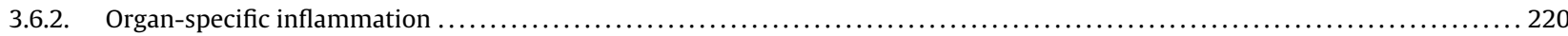

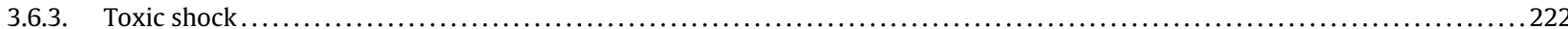

\footnotetext{
* Corresponding author at: Bldg 567 Rm 205, Frederick National Laboratory for Cancer Research, Frederick, MD 21702, USA. Fax: +1 302 1184281.

** Corresponding author at: Biotherapeutic ODN Research Laboratory, Faculty of Sciences, Bilkent University, Bilkent, Ankara 06800 , Turkey. Fax: +90 3122665097.

E-mail addresses: ihsangursel@bilkent.edu.tr (I. Gursel), klinmand@mail.nih.gov (D.M. Klinman).
} 


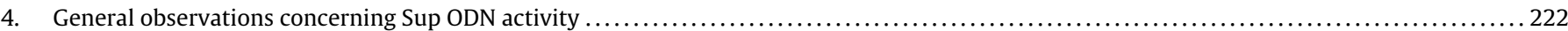

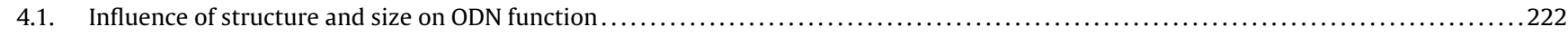

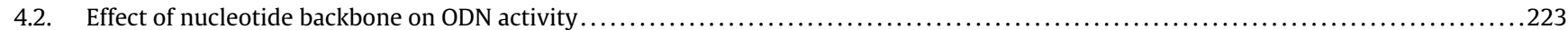

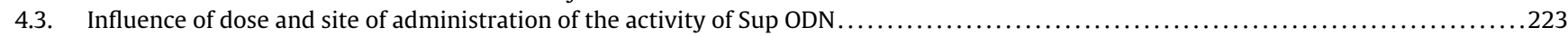

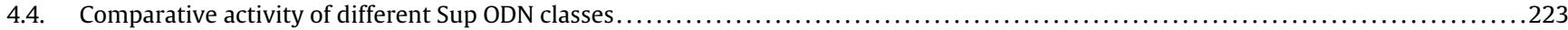

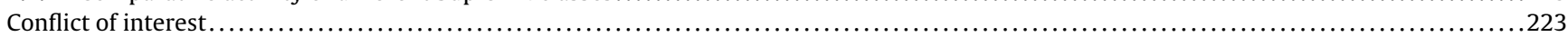

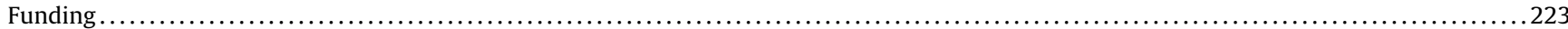

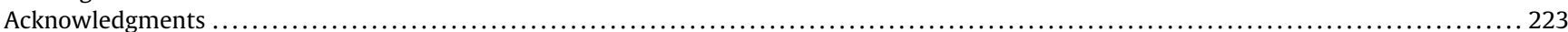

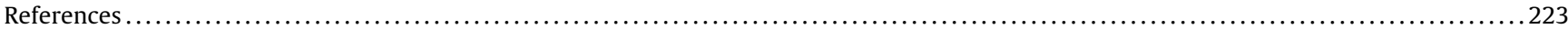

\section{Introduction}

Nucleic acids are the "blueprint of life" and thus essential components of all living organisms. DNA and RNA also have multiple and complex effects on the immune system [1-3]. The nucleic acids present in pathogenic microorganisms can trigger toll-like receptors on immune cells, stimulating them to mount a protective response [4-9]. Conversely, the telomeres that cap mammalian DNA contain repetitive TTAGGG motifs that inhibit immune reactions [1]. The release of inhibitory DNA as host cells die may serve to down-regulate pathologic inflammatory and autoimmune responses. This work reviews the use of synthetic oligonucleotides containing immunosuppressive motifs (Sup ODN) for the treatment of cancer, inflammation and autoimmune disorders.

\subsection{Historical overview}

The ability of DNA to inhibit immune reactions was first observed in studies of phosphorothioate modified ODN. ODNs (particularly those expressing poly-G motifs) suppressed IFN production by activated murine splenocytes. However neither the precise sequence nor mechanism of action underlying that suppressive activity was carefully investigated. Indeed, 30-mers of widely varying sequence were reported to mediate various degrees of immune suppression [10-12].

In 1998, Krieg et al. reported that DNA from certain adenovirus serotypes contained in "immunoinhibitory" motifs that could down-regulate TLR-induced immune activation [13]. Other suppressive motifs were subsequently described, some of which blocked other forms of immune activation as well [1,14-18]. Much of this activity was linked to the presence of extended $G$ and C-rich sequence motifs [13,19]. In studies of mammalian DNA, Gursel et al. found that immune suppression was largely mediated by the repetitive TTAGGG motifs present in mammalian telomeres [1]. G-rich and microsatellite regions were later found to further contribute to the suppressive activity of mammalian DNA [20]. Of interest, the genomes of immunomodulatory commensal bacteria are now known to contain suppressive DNA motifs [16].

Oligodeoxynucleotides that mimic the immunosuppressive activity of mammalian DNA (referred to hereafter as "Sup ODN") were synthesized and tested by many groups. As described below, these vary in sequence and mechanism of action. Several groups sought to categorize these different types of Sup ODN. Trieu et al. proposed grouping them into four classes based on their sequence and probable mode of action [21] whereas Lenert categorized them based on their ability to form secondary structures (including $G$ tetrads and palindromes) [22]. This review describes the sequence, mechanism of action and therapeutic potential of multiple classes of Sup ODN that are categorized based on the breadth of their inhibitory activity. Broadly acting Sup ODNs act on multiple cell types and suppress the immune activation elicited by many different stimulants. By comparison, TLR-specific Sup ODN primarily antagonize TLR9 and/or TLR7 induced responses and their activity is limited to cells expressing those receptors.

\section{Broadly acting Sup ODN}

\subsection{Mechanisms of action}

A151 is the archetypal example and best studied of the broadly acting Sup ODN. A151 is composed of four TTAGGG motifs designed to mimic the repetitive elements present at high frequency in mammalian telomeres. Telomeric DNA inhibits the activation and differentiation of macrophages, dendritic cells, B cells and multiple subsets of T cells [1,16,18,23-27].

A151 blocks the immune stimulation induced by bacterial DNA, an effect initially attributed to competition for binding between A151 and CPG ODN to TLR9. Subsequent research showed that the broad immunosuppressive activity of A151 was primarily attributable to its effect on STAT phosphorylation. STAT proteins are transcription factors that influence the maturation of many types of immune cell (reviewed in Ref. [28]). Evidence that A151 interfers with the phosphorylation of STAT1 and STAT4 was obtained in studies of TLR4-stimulated macrophages [23]. Inhibition of STAT3 phosphorylation was then observed in studies of naive CD4 T cell differentiation. A151 binds to STATs 1,3 and 4 to inhibit downstream signaling, thereby inhibiting the production of IFNg and IL-12 which interferes with the generation of proinflammatory Th1 lymphocytes. This skews the cytokine milieu and supports the generation of Th2 responses in vivo [24].

The effect of A151 on STAT phosphorylation pre-dated the discovery of Th17 and regulatory T cells (Treg) whose influence on the development of autoimmune and inflammatory diseases is now appreciated. A151 supports the generation of Th17 cells by blocking the generation of SOCS3, a negative regulator of phosphoSTAT3 [15]. A151 also promotes the generation of Tregs. This arises from a direct effect of A151 in blocking STAT1 phosphorylation which enables naive $\mathrm{CD} 4^{+} \mathrm{CD} 25^{-} \mathrm{T}$ cells to differentiate into $\mathrm{CD} 4^{+} \mathrm{CD} 25^{+} \mathrm{FoxP}^{+}$iTregs and an indirect effect whereby $\mathrm{A} 151$ interferes with the generation of LpDC that would otherwise reduce Treg generation $[16,27]$. Studies of human B cells indicates that A151 can suppress B cell activation, Ab production and the generation of plasma/memory cells [18]. This activity is attributed to the ability of A151 to suppress AICDA (activation induced cytidine deaminase) which is known to regulate class switch and somatic mutation in B cells [29] (Fig. 1).

An additional target of A151 was recently described. AIM2 and IFNg-inducible protein-16 (IFI16) are DNA-binding proteins that recognize cytosolic bacterial and viral dsDNA. Activation of these proteins recruits caspase- 1 to mediate the cleave of pro-IL-1B and pro-IL-18 into their functional forms [30-32]. A151 directly binds to AIM2 which prevents the recruitment of ASC and the subsequent assembly of the inflammasome complex [26]. Thus, the ability of A151 to broadly suppress the differentiation and activation of 


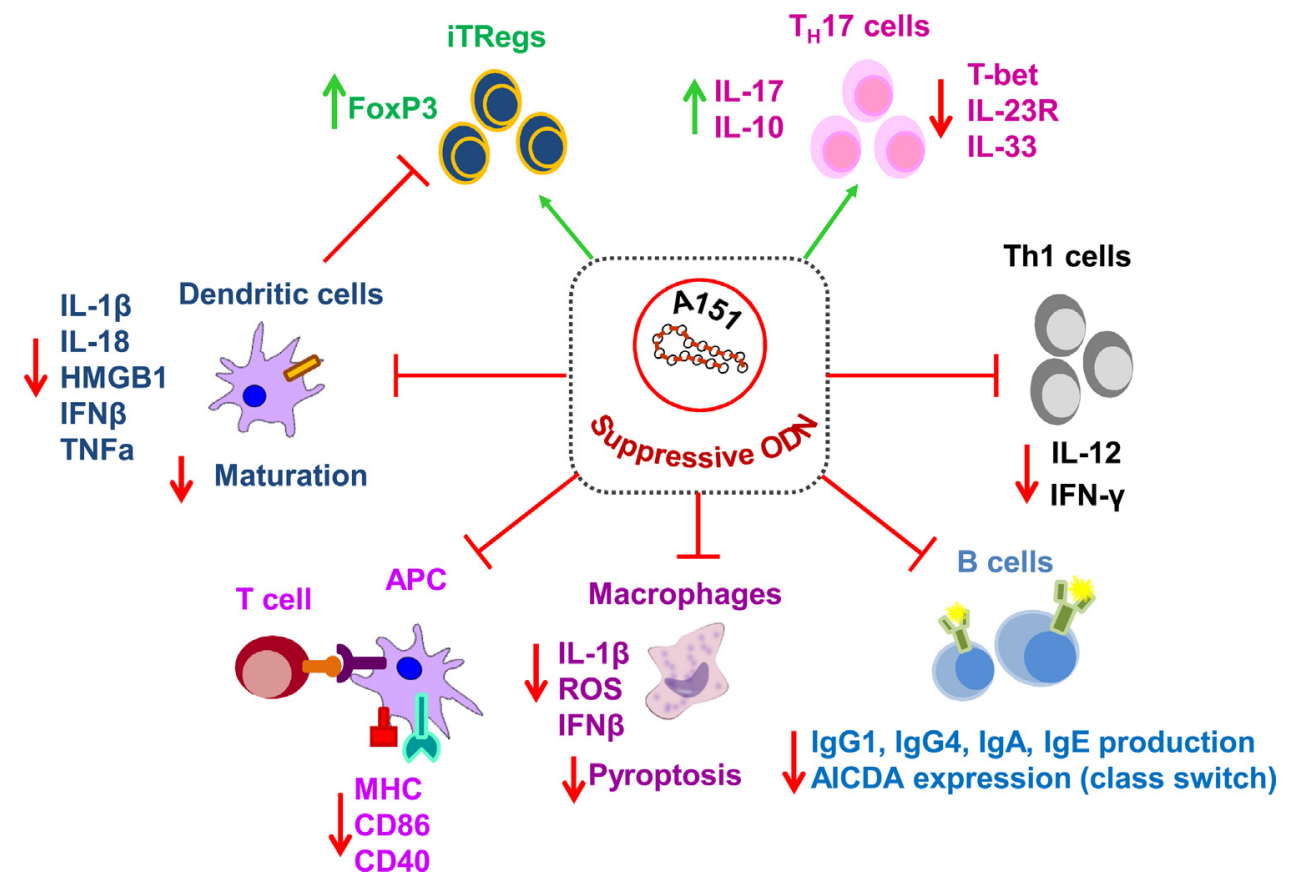

Fig. 1. Suppressive ODN A151 has diverse effects on cellular elements of the immune system.

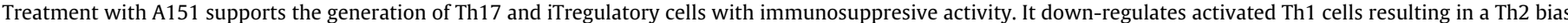

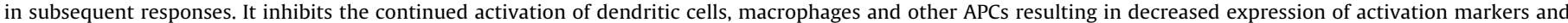
reduced production of proinflammatory cytokines. B cell maturation, class switching and Ig production are also suppressed.

multiple cell types derives from its ability to act on multiple signaling pathways (Fig. 1).

\subsection{Therapeutic activity}

\subsubsection{A151 for the treatment of autoimmune and infectious diseases}

The first therapeutic uses of Sup ODN were for the prevention and/or treatment of autoimmune diseases. As these studies were performed over a decade ago and have already been reviewed [33], only a brief overview of salient findings is provided below.

1) Lupus. The effect of delivering A151 to lupus-prone NZB/W mice was examined in this spontaneous disease model both before and after immune complex mediated kidney damage had developed. Early treatment slowed the onset and reduced the magnitude of autoimmune-mediated renal inflammation leading to significantly prolonged survival [34]. Starting treatment after mice were already sick slowed but did not prevent disease progression.

2) Arthritis. Intra-articular delivery of A151 significantly reduced the incidence and severity of arthritis in an animal model of collagen-induced arthritis. This treatment also decreased serum titers of pathogenic IgG autoAb [14].

3) Autoimmune uveitis. Three different models of autoimmune uveitis were examined: acute, recurrent, and persistent. In each case, treatment with A151 significantly reduced the magnitude of ocular inflammation and subsequent tissue damage $[3,35,36]$.

4) Atopic dermatitis. A recent report by Wang et al. broke new ground in the therapeutic use of Sup ODN by showing they can be delivered orally to treat skin disease. A151 was encapsulation in acid stable nanoparticles to protect them from degradation in the stomach. After oral delivery the nanoparticles reached the small intestine where they were selectively taken up by macrophages in the Peyers patches. Repeated delivery resulted in systemic changes in cytokine production (reducing levels of IL
4 and IL 33) and reduced the differentiation of allergen-activated Th2 cells thereby attenuating the development of chemically induced atopic dermatitis [17].

\subsubsection{A151 for the treatment of toxic shock}

Toxic shock arises from the cytokine storm triggered by overwhelming bacterial sepsis. This effect can be replicated by the delivery of high dose LPS to normal mice. Treatment with A151 at the same time as LPS challenged significantly reduced cytokine storm and improved survival [23]. The longer therapy was delayed, the less effective it became.

\subsubsection{A151 for the treatment of organ specific inflammation}

Studies of A151 document the ability of this class of ODN to ameliorate organ-specific inflammation. Pulmonary inflammation was evaluated in a murine model of silicosis. Similar to coal dust and asbestos, inhalation of silica particles causes progressive fibrosis, reduced blood oxygenation, and increased susceptibility to cancer [37]. Silicosis is elicited in mice by instilling silica particles into the lungs which causes an inflammatory infiltrate, increased production of pro-inflammatory cytokines and chemokines (including IL-6, IL-1, TNFa, IL-12 and keratinocyte chemoattractant (KC)), and alveolar hemorrhage (reviewed in Ref. [38]). Treating mice with A151 shortly before silica instillation significantly reduced cellular infiltration of the lungs and local production of pro-inflammatory cytokines [25]. Of clinical relevance, A151 prevented silica-induced weight loss and significantly improved survival. Thus, A151 both reduced local inflammation and ameliorated systemic symptoms. However treatment was ineffective if delayed until chronic silicosis had developed.

Inflammation of the GI tract has been shown to contribute to the development of autoimmune disease and cancer (reviewed in Ref. [39]). The effect of Sup ODN treatment in two different murine models of gut inflammation was examined. The first involved infection with the Toxoplasma gondii parasite and the second topical exposure to the chemical irritant dextran sulfate (DSS). In both 
systems, repeated oral delivery of A151 down-regulated the production of pro-inflammatory cytokines (IFNg, TNFa, IL-6 and IL-22) and maintained the integrity of the gut epithelium [16]. This was linked to the ability of A151 to down-regulate LpDC activation, thereby maintaining IL-10 production and sustaining Treg activity [16]. Independent studies showed that A151 directly supported the generation of Tregs [27]. Together these findings indicate that A151 supports gut homeostasis by maintaining Treg function that would otherwise be dysregulated in inflammatory bowel disease (reviewed in Ref. [40]).

\subsubsection{A151 for the treatment of fungal infection}

Although healthy individuals rarely suffer from major fungal infections, some fungal strains are pathogenic particularly in immunosuppressed hosts [41]. Recent evidence suggests Th17 immunity plays an important role in clearing fungal infections [35]. In vitro studies showed that A151 promoted the generation of Th17 cells by inhibiting SOCS3 which is a negative regulator of Th17 differentiation [15]. Using Candida albicans as a model pathogen, the ability of A151 to generate Th17 cells capable of protecting against fungi was examined in mice. Results showed that systemic treatment with A151 increased Th17 immunity and that this was associated with reduced weight loss and a lower infectious burden in C. albicans challenged animals when compared to untreated controls [15].

\subsubsection{A151 for the treatment of atherosclerosis}

Atherosclerosis is characterized by the deposition of plaque (composed of macrophages, fat, cholesterol and calcium) in the arteries. Advanced atherosclerosis increases the risk of myocardial infarction, peripheral vascular disease and stroke. Inflammation is an important component of the atherosclerotic process. Activated $\mathrm{T}$ cells produce factors that stimulate macrophages to internalize lipoproteins and become artery-occluding foam cells. ApoeE KO mice are widely used to model this atherosclerotic process [42]. These animals rapidly develop extensive plaques associated with markers of atherosclerotic inflammation including MCP-1 and VCAM-1.

The effect of treating ApoE mice with A151 was evaluated. Serum levels of MCP-1 and VCAM fell by $30-50 \%$ ( $p<.05$ for both factors) while the size of the atherosclerotic lesions was reduced by half [43]. Levels of the Th1 cytokines IFNg and TNFa were significantly reduced, an effect that correlated with reduced size of the atherosclerotic lesions. Mechanistically, Sup ODN treatment reduced the phosphorylation of STAT1 and STAT4 thereby reducing the T-bet expression needed to support Th1 cell differentiation. As a result, the frequency of IFNg production Th1 cells declined while the ratio of Th2:Th1 cells rose.

\subsubsection{A151 for the treatment of stroke}

The ability of Sup ODN to prevent ischemic stroke was examined using stroke-prone hypertensive (SHR-SP) rats. Stroke is a major cause of chronic debilitation and the second most common cause of death worldwide. While strokes are caused by a reduction in blood supply to the brain, the resulting tissue damage triggers an inflammatory response that further increases lesion size [44-46].

Zhao et al. examined the effect of treatment with A151 on strokes generated by surgically occluding the middle cerebral artery of SHR-SP rats [47]. Results indicate that A151 had broad anti-inflammatory properties, associated with decreased production of caspase-1, IL-1ß, iNOS and NLRP3 by activated macrophages. Sup ODN treatment limited the magnitude of ischemia-induced brain damage in a time and dose dependent fashion. A151 was most effective when administered 1 day prior to infarct induction. The highest dose tested $(3 \mathrm{mg}$ ) was more effective than $1 \mathrm{mg}$. Under optimal conditions, Sup ODN reduced the extent of brain damage by $>25 \%$ [47]. These observations are relevant to patients scheduled to undergo cardiac or carotid surgery whose high risk of stroke may be reduced by treatment with Sup ODN prior to surgery.

\subsubsection{A151 for the prevention of inflammation-induced cancer}

Chronic inflammation contributes to the development and progression of many types of cancer (reviewed in Ref. [48]). The possibility that Sup ODN might interfere with the inflammation that supports tumorigenesis was therefore explored. The first study in the field focused on a common murine model of skin cancer in which TPA was used to drive inflammation after transformation was initiated by DMBA. Mice treated with DMBA/TPA typically develop skin papillomas that transform into squamous cell carcinomas (SCC) over time [49].

Ikeuchi et al. examined the effect of administering A151 at the same time as TPA. Results showed that Sup ODN therapy reduced papilloma formation by $95 \%$ and that this effect was dosedependent. Histological analyses revealed that A151 limited the development of edema, leukocyte infiltration and the production of various markers of inflammation (including CCL2, CXCL2, COX2 and ornithine decarboxylase) [50]. Discontinuing or delaying the initiation of Sup ODN therapy slowed but did not prevent papillomas from arising [50].

A large body of data suggests that pulmonary inflammation increases the risk of cigarette smoke induced lung cancer (reviewed in Ref. [51]). To evaluate whether A151 could alter susceptibility to lung cancer by reducing inflammation, a murine model was developed in which NNK (a highly carcinogenic component of cigarette smoke) was delivered to mice with silica-induced pulmonary inflammation. The combination of NKK plus silica increased the fraction of mice that developed lung tumors (incidence) and the number of tumors per mouse (multiplicity) [52]. Treating these mice with A151 starting at the time of silica administration reduced pulmonary inflammation as evidenced by a significant decrease in macrophage and neutrophil infiltration, lower levels of pro-inflammatory cytokines (including IL-1B and TNFa) and less fibrosis [52]. Treatment with A151 also reduced to background the incidence and multiplicity of lung tumors in NNK-treated silicotic mice. Additional studies showed that A151 improved the anti-proliferative effects of several chemotherapeutic drugs [53]. These results strongly suggest that Sup ODN may help prevent inflammation-driven cancers from developing.

\section{TLR specific Sup ODN}

\subsection{Mechanism of action}

A variety of Sup ODN function by selectively blocking the effects of TLR9 and/or TLR7 agonists. Various types of TLR-specific Sup ODN act on different stages of the TLR signaling cascade: some compete for uptake, others inhibit receptor binding and/or block downstream signaling. The following represents an overview of the effects of these types of Sup ODN.

\subsection{H154 sequence: $5^{\prime}$-CCTCAAGCTTGAGGGG-3'}

H154 is a specific inhibitor of the immune activation induced via TLR9. H154 interferes with downstream signaling rather than by inhibiting the binding or uptake of CpG DNA [8]. This results in a significant reduction in cytokine and $\mathrm{Ab}$ production by cells activated via TLR9 $[8,54]$. Reflecting its specificity for TLR9, H154 cannot downregulate immune responses triggered by other immune stimulants such as LPS or ConA [8]. Thus, while effective for treating inflammatory conditions triggered via TLR9 the therapeutic utility of H154 is more limited than that of A151 [55-57]. 


\subsection{Inhibitory (INH) ODNs (e.g., TCCTGGCGGGGAAGT)}

INH ODNs selectively interfere with TLR9-mediated immune activation by competing with $\mathrm{CpG}$ ODN for binding to the Cterminal region of TLR9 $[58,59]$. Most INH ODNs have sequences similar to CpG ODN with the critical difference that the receptor activation residues are absent [60-62]. The interaction of INH ODN with TLR9 fails to induce the conformational changes necessary for activation of the downstream signaling cascade via MyD88 with the result that NF-kB and AP1 activation never occurs [59,63-65]. Cells that express TLR9, including B cells, dendritic cells and murine monocytes, are all inhibited by INH ODN. INH ODNs are also reported to down-regulate TLR7-mediated immune activation to some extent, although that effect may not be sequence specific [66-68].

There is limited evidence that INH ODN might increase host susceptibility to bacterial infection. The gram negative bacterium Salmonella typhimurium replicates within macrophages and is a common cause of food-borne illnesses [69]. Independent of any effect on TLR9 signaling, INH ODN increase bacterial load in macrophages due to partial inhibition of TLR1/2 signaling, a sideeffect that might alter the host-microbe response [70].

\section{4. 'G' ODN}

' $G$ ' ODN contain a string a five guanines with a representative sequence being CTCCTATTGGGGGTTTCCTAT. ' $G$ ' ODN bind to the C-terminal region of TLR9 thereby preventing CpG-receptor interaction [59]. As a result, this class of ODN dampens TLR9 mediated activation of APC and the production of pro-inflammatory cytokines including IFNa, TNFa and IL-12 [71].

\subsubsection{Modified ODNs}

Modified ODNs are generated by reversing stimulatory $\mathrm{CpG}$ motifs to $\mathrm{GpC}$ or GpG. While their mechanism of action has not been clarified, their sequence similarity to CpG ODN strongly suggests that competition for uptake, binding and/or receptor activation underllies their activity. Sequences such as 5'-TGACTGTGAAGGTTAGAGATGA-3' antagonize CpG-mediated immunity by limiting the activation of APC and production of proinflammatory cytokines [72]. In various in vivo models, GpG ODNs support Th2 rather than Th1 responses, an effect accompanied by decreased production in pro-inflammatory cytokines [73,74].

An atypical example of this class of ODN is GpC-1826. GpC-1826 utilizes the TLR7/TRIF signaling pathway to increase indoleamine 2,3-dioxygenase (IDO) expression thereby producing tolerogenic pDCs $[75,76]$. A modified version of this ODN supported the generation of Treg indirectly by promoting tolerogenic pDC [77]. GpC-1826 antagonizes immune response mediated by TLR7 agonists while its effect on responses elicited via TLR9 is unknown.

A different mechanism of action was described for GpC-1668 and GpG-1668. These mediate immune suppression by binding to high-mobility group box proteins (HMGBs) [78]. HGMBs are essential for the recognition of nucleic acids that trigger receptor mediated immune responses [78]. By competing with stimulatory nucleic acids for intracellular HMGB, these Sup ODN inhibit dsDNA, ssDNA and dsRNA-mediated immune activation.

\subsection{Sup ODNs whose mechanisms of action has not been established}

Several groups described novel ODNs with inhibitory activity but failed to examine the mechanism through which they blocked immune responses. Immunoregulatory DNA sequences (IRS) and microsatellite sequences are examples of such ODNs.
IRS 869 (TGCTCCTGGAGGGGTTGT) is a G-rich TLR9 antagonist that prevents TLR9-mediated endotoxic shock by blocking the release of pro-inflammatory cytokines [79]. Given the similarity in sequence between IRS 869 and INH ODNs, it is likely that this effect is mediated by competition for binding with CPG DNA to TLR9. IRS 661 blocks TLR7 signaling while IRS 954 down-regulates responses elicited by both TLR7 and TLR9 agonists [73]. While inhibition by IRS ODN was observed in multiple cell types of mouse and human origin, no information was provided on whether their activity involved competition at the uptake/receptor-binding level or modulation of downstream signaling.

Other groups evaluated 24-mer ODNs consisting of multiple TC, AAAG or CCT repeats and reported that several impaired IFN production by human PBMC $[20,80]$. Similar sequences are present in a subset of human microsatellite regions, leading the investigators to name them microsatellite (MS) ODN.

However no evidence that human microsatellites are immunosuppressive has been provided. MS08, a prototypic MS ODN, blocks the uptake of CpG ODNs and thus suppresses TLR9 mediated immune activation. However MS08 also down-regulates TLR independent immune responses although no underlying mechanism was identified [20]. Other MS ODNs vary in their ability to influence $\mathrm{CpG}$-induced inflammation and discrepancies exist between the in vitro vs in vivo activity of this class of ODN, raising uncertainty over their potential therapeutic utility $[20,80,81]$.

\subsection{Therapeutic activity}

\subsubsection{Autoimmune disease}

1) In a murine model of reactive arthritis (an inflammatory condition triggered by bacterial infection), Zeuner et al. showed that injecting $\mathrm{H} 154$ into an affected joint significantly reduced both inflammation and swelling. Since arthritis can affect multiple joints, H154 ODN was also administered i.p. and found to reduce systemic inflammatory arthritis [56,57].

2) In the NZB $\times$ NZW F1 mouse model of lupus, GpG ODN treatment promoted Th2 biased immune responses that delayed the onset of proteinuria [74]. Treatment with IRS 661 and 954 reduced serum anti-nuclear $\mathrm{Ab}$ levels, the deposition of immune complexes in the kidneys and delayed disease progression [67,79,82-87]. In lupus prone MRL lpr/lpr mice, INH ODNs suppressed autoreactive B cell and DC responses leading to reduced autoantibody production $[22,66]$. In a murine model of lupus induced by chronic graft versus host disease, He et al. reported that MS ODNs and Sat05f reduced anti ssDNA antibody levels and delayed disease progression [88].

3) In the EAE model of multiple sclerosis, adding GpC ODN to a toleragenic DNA vaccine reduced disease severity by inducing autoreactive $B$ and $T$ cell responses to shift to a protective IgG1 isotype and Th2 type cytokine pattern [72,73]. In those studies, Sup ODN competed with CpG sequences in the vaccine to inhibit Th1 responses.

4) Experimental autoimmune neuritis provides a model of Guillain Barre Syndrome characterized by demyelination and inflammation of the peripheral nervous system. It is induced by injecting P2 peptide in complete Freund's adjuvant into the hind footpads of Lewis rats. When animals with EIN were treated with H154, markers of inflammation and disease severity were significantly reduced [89].

\subsubsection{Organ-specific inflammation}

In a murine model of acute lung inflammation, MS19 significantly inhibited weight loss and hemorrhage, reduced intraalveolar edema and lessened the accumulation of neutrophils in the lungs $[81,88,90]$. H154 inhibited the pulmonary inflammation induced by the delivery of immunostimulatory bacterial products 
Table 1

Overview of suppressive oligonucleotides.

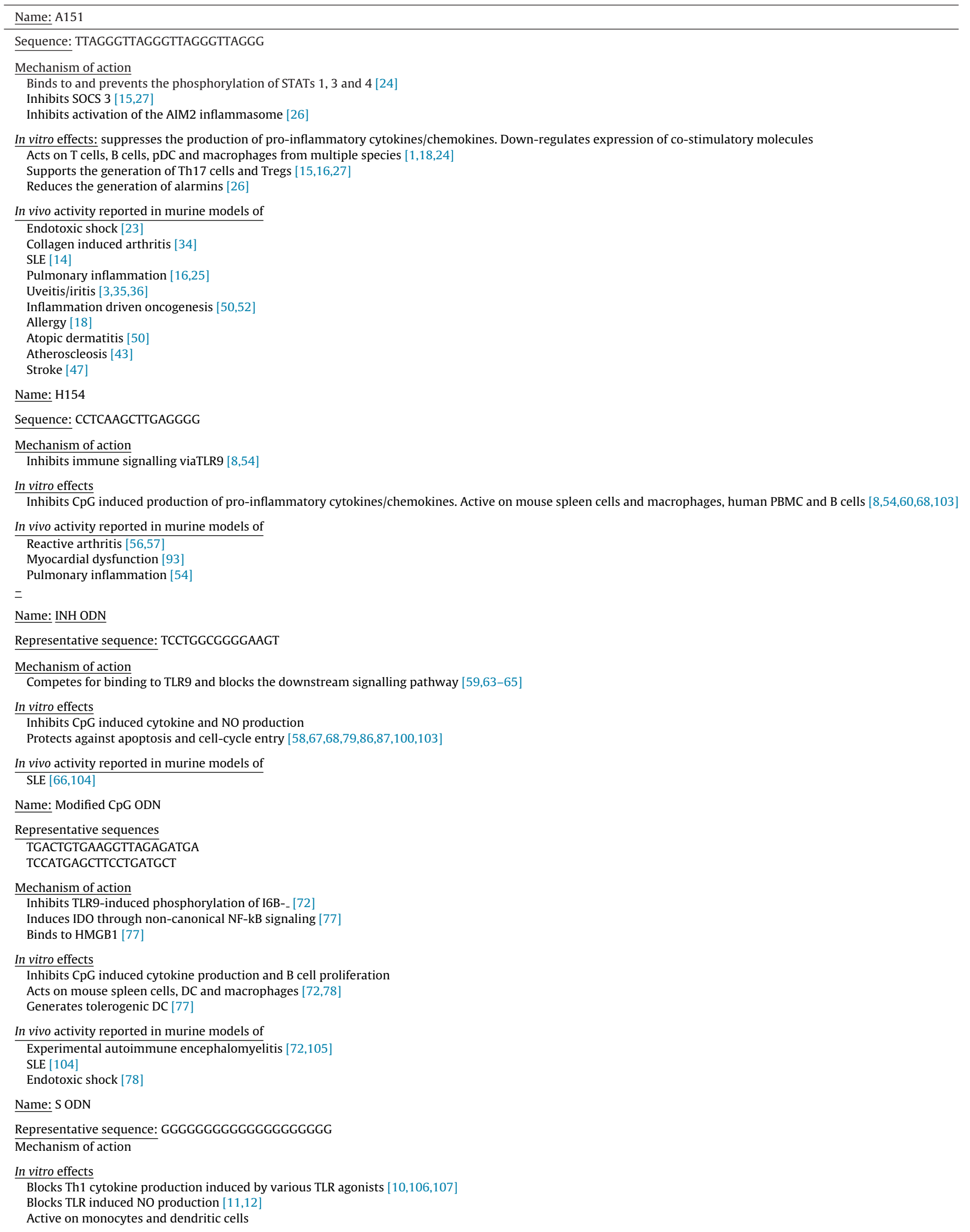


Table 1 (Continued)

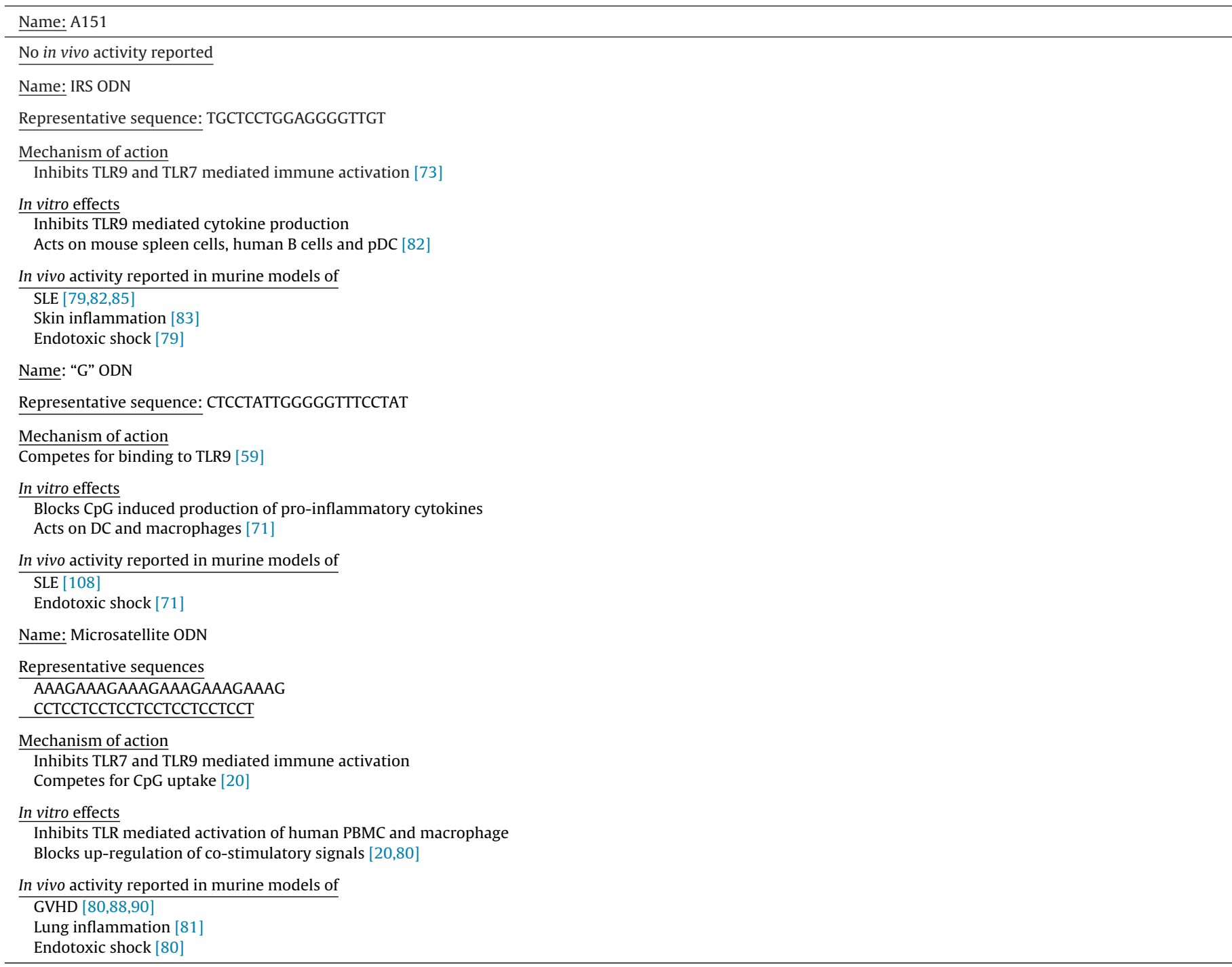

(such as CpG DNA) into the lungs of mice was evaluated [91]. CpG instillation triggered a local response characterized by neutrophil accumulation and increased TNFa, IL-6, MIP-2, and KC production. Co-delivery of Sup ODN H154 significantly lessened the magnitude of these inflammatory changes [54].

In a murine model of myocardial dysfunction elicited by activation of TLR9, Boehm et al. found that ODN H154 significantly ameliorated cardiac inflammation, preserved cardiac function, and improved survival [92,93].

\subsubsection{Toxic shock}

MS ODNs and Sat05f inhibited TLR7 and TLR9 mediated innate immune responses thereby protecting mice from D GalN/CpG ODN induced lethal shock [81].

'G' ODN protected mice from cytokine-mediated lethal shock induced by bacterial DNA [71]. GpG 1668 protected against LPS-induced toxin shock by reducing the production of proinflammatory cytokines. While this effect was attributed to HMGB targeting, it should be noted that $\mathrm{GpG}-1668$ was unable to downregulate LPS-induced immune responses in vitro.

\section{General observations concerning Sup ODN activity}

\subsection{Influence of structure and size on ODN function}

While distinct classes of Sup ODN differ in length, sequence and functional activity, most contain a string of poly-Gs $[1,8,62,64,71,79]$. Suppressive activity typically requires a minimum of 3 G's, with several studies suggesting that longer runs of poly-G increase potency further [58,60,62,79]. Conversely, reducing the number of G's typically reduces or ablates suppressive activity $[1,26,94]$.

Poly-G sites enable the formation of higher order quadruplex structures via inter-chain Hoogsteen hydrogen bonding (reviewed in Ref. [95]). This binding is disrupted by insertion of a 7deazaguanine (7-DG) nucleotide which prevents hydrogen bond formation but does not affect Watson-Crick pairing [96]. In studies of INH ODN, monomeric structures (generated by substituting 7-DG for one or more G's) remained functional, indicating that quadruplex formation was not required for their inhibitory activity $[58,67,68,79,97]$. In contrast, the ability to form G-tetrads was required for A151 to its maintain broad immunosuppressive activity since substituting a 7-DG for any G significantly reduced inhibitory function [1]. 
This difference in the role of quadruplex structures may distinguish between ODN that act in a TLR-specific versus broadly suppressive manner. Whereas single stranded ODN might effectively compete with single-stranded CpG ODN for binding to TLR9, quadruplex structures may be necessary to facilitate the interaction of A151 with molecular targets including STATs and inflammasome components. In this context, G-tetrads are known to make a critical contribution to the binding of ODN to STAT3, an important target of A151 [98].

Length also influences the activity of Sup ODN. A single TTAGGG 6 -mer has no activity yet the same motif conjugated to a random 8-mer exhibits suppressive activity [1]. Similarly, a 5-mer poly-G is suppressive only when incorporated into a longer ODN [71]. Studies of various classes of Sup ODN indicate that sequences shorter than 11 nucleotides have little suppressive activity while those longer than 24 nucleotides gain little additional function $[8,62,72,77,78,90]$. This pattern was also observed in studies of TTAGGG multimers: suppressive activity increased as more motifs were added but only to a point, with ODN containing 5 repeats being no more active than those with 4 TTAGGG repeats [1,90].

\subsection{Effect of nucleotide backbone on ODN activity}

Native DNA is composed of nuclease-sensitive phosphodiester (PO) base pairs that are rapidly degraded in vivo. To improve therapeutic half life, the non-bridging oxygen can be replaced with sulfur to yield phosphorothioate (PS) modified ODN. PS are superior to PO ODN in terms of both nuclease resistance and cellular uptake (reviewed in Ref. [99]). The potency of PS vs PO was examined for several classes of Sup ODN. In vitro studies show that A151PS and A151-PO are equally efficient in suppressing CpG induced responses whereas only A151-PS was much more effective in blocking dsDNA-induced inflammasome activation in vivo $[1,26]$. Other studies confirmed the superior potency of PS over PO versions of the same Sup ODN in vivo $[71,78,79,100]$. It should be noted that sequence-independent inhibition of immune responses has been reported for some PS ODNs [10,11,66,101].

\subsection{Influence of dose and site of administration of the activity of Sup ODN}

In vitro studies by many groups establish that Sup ODN can inhibit the production of pro-inflammatory cytokines and chemokines (including IL6, IL-12, IFNg, TNFa and MIP2a) $[1,12,24,33]$. These effects are summarized in Table 1 . We draw the following general conclusions from analysis of multiple autoimmune and inflammatory disease models.

1) Sup ODN are most effective when administered immediately prior to or concomitant with the delivery of the inflammatory stimulus $[3,102]$. This is consistent with evidence that Sup ODN effectively block the activation of inflammatory immune cells but are relatively ineffective at down-regulating cells that have already been activated [8].

2 ) The effect of Sup ODN is dose and location dependent. In studies where A151 was delivered systemically, the effective dose in mice was typically $300 \mu \mathrm{g}[3,34]$. However much lower doses were sufficient when A151 was delivered locally. For the treatment of arthritis, as little as 10 ug injected into the knee was sufficient whereas 30-50 $\mu \mathrm{g}$ prevented pulmonary inflammation $[25,54]$.

\subsection{Comparative activity of different Sup ODN classes}

Very few studies have compared the activity of different classes of Sup ODN. Those comparisons that were conducted generally used in vitro assays to examine a single immune parameter and cell type and thus are unlikely to reflect broad in vivo efficacy. For example, experiments indicate that INH ODN 2114 and H154 are equivalent in terms of stimulating cytokine production in vitro but that $\mathrm{H} 154$ is a less potent suppressor of B cell activation and proliferation $[60,62,103]$. Other studies focusing on B cell activation suggest that INH ODN 2114 is superior to 'G' ODN but inferior to IRS954 and IRS869 [103]. A151 and microsatellite ODN have similar capacities to block PBMC proliferation and pro-inflammatory cytokine production $[20,80]$. Another study revealed that INH ODN 2114 and A151 inhibited CpG-driven NF-kB up-regulation in macrophage to the same degree [21]. Lacking adequate in vivo comparisons, the extent and breadth of immune suppression mediated by A151 marks it as a superior candidate for clinical development.

\section{Conflict of interest}

Dr. Dennis Klinman and members of his lab are co-inventors on a number of patents concerning Sup ODN and their use. All rights to these patents have been assigned to the Federal government.

\section{Funding}

This research was supported by the Intramural Research Program of the National Cancer Institute of the National Institutes of Health.

\section{Acknowledgments}

This manuscript was supported by the Intramural Research Program of the $\mathrm{NIH}, \mathrm{NCI}$. The content of this publication does not necessarily reflect the views or policies of the Department of Health and Human Services, nor does mention of trade names, commercial products, or organizations imply endorsement by the United States government. The funders had no role in study design, data collection and analysis, decision to publish, or preparation of the manuscript.

\section{References}

[1] I. Gursel, M. Gursel, H. Yamada, K.J. Ishii, F. Takeshita, D.M. Klinman, Repetitive elements in mammalian telomeres suppress bacterial DNA-induced immune activation, J. Immunol. 171 (2003) 1393-1400.

[2] K.J. Ishii, S. Akira, Innate immune recognition of nucleic acids: beyond toll-like receptors, Int. J. Cancer 117 (2005) 517-523.

[3] F.C. Yagci, O. Aslan, M. Gursel, G. Tincer, Y. Ozdamar, K. Karatepe, et al., Mammalian telomeric DNA suppresses endotoxin-induced uveitis, J. Biol. Chem. 285 (2010) 28806-28811.

[4] S. Yamamoto, T. Yamamoto, S. Shimada, E. Kuramoto, O. Yano, T. Kataoka, et al., DNA from bacteria, but not vertebrates, induces interferons: activate NK cells and inhibits tumor growth, Microbiol. Immunol. 36 (1992) 983-997.

[5] D.M. Klinman, A. Yi, S.L. Beaucage, J. Conover, A.M. Krieg, CpG motifs present in bacterial DNA rapidly induce lymphocytes to secrete IL-6, IL-12 and IFNg, Proc. Natl. Acad. Sci. U. S. A. 93 (1996) 2879-2883.

[6] T. Sparwasser, E. Koch, R.M. Vabulas, K. Heeg, G.B. Lipford, J.W. Ellwart, et al., Bacterial DNA and immunostimulatory CpG oligonucleotides trigger maturation and activation of murine dendritic cells, Eur. J. Immunol. 28 (1998) 2045-2054.

[7] A.M. Krieg, The role of $\mathrm{CpG}$ motifs in innate immunity, Curr. Opin. Immunol. 12 (2000) 35-43.

[8] H. Yamada, I. Gursel, F. Takeshita, J. Conover, K.J. Ishii, M. Gursel, et al., Effect of suppressive DNA on CpG-induced immune activation, J. Immunol. 169 (2002) 5590-5594

[9] D.M. Klinman, S. Klaschik, T. Sato, D. Tross, CpG oligonucleotides as adjuvants for vaccines targeting infectious diseases, Adv. Drug Deliv. Rev. 61 (2009) 248-255.

[10] D.S. Pisetsky, C.F. Reich, Inhibition of murine macrophage IL-12 production by natural and synthetic DNA. Clin. Immunol. 96 (2000) 198-204.

[11] F.G. Zhu, C.F. Reich, D.S. Pisetsky, Inhibition of murine macrophage nitric oxide production by synthetic oligonucleotides 9, J. Leukoc. Biol. 71 (2002) 686-694. 
[12] F.G. Zhu, C.F. Reich, D.S. Pisetsky, Inhibition of murine dendritic cell activation by synthetic phosphorothioate oligodeoxynucleotides, J. Leukoc. Biol. 72 (2002) 1154-1163.

[13] A.M. Krieg, T. Wu, R. Weeratna, S.M. Efler, L. Love, L. Yang, et al., Sequence motifs in adenoviral DNA block immune activation by stimuatory CpG motifs, Proc. Natl. Acad. Sci. U. S. A. 95 (1998) 12631-12636.

[14] L. Dong, S. Ito, K. Ishii, D. Klinman, Suppressive Oligodeoxynucleotides Delay the Onset of Glomerulonephritis and Prolong the Survival of Lupus-prone NZB/W Mice, Arthritis Rheum. 52 (2004) 651-658.

[15] C. Bode, X.P. Yang, H. Kiu, D.M. Klinman, Suppressive oligodeoxynucleotides promote the development of Th17 cells, PLoS One 8 (2013) e67991.

[16] N. Bouladoux, J.A. Hall, J.R. Grainger, L.M. dos Santos, M.G. Kann, V. Nagarajan, et al., Regulatory role of suppressive motifs from commensal DNA, Mucosal Immunol. 5 (2012) 623-634.

[17] Y. Wang, Y. Yamamoto, S. Shigemori, T. Watanabe, K. Oshiro, X. Wang, et al., Inhibitory/suppressive oligodeoxynucleotide nanocapsules as simple oral delivery devices for preventing atopic dermatitis in mice, Mol. Ther. 23 (2015) 297-309.

[18] C. Sackesen, V. van d, M. Akdis, O. Soyer, J. Zumkehr, B. Ruckert, et al., Suppression of B-cell activation and IgE, IgA: IgG1 and IgG4 production by mammalian telomeric oligonucleotides, Allergy 68 (2013) 593-603.

[19] Q.Zhao, J. Temsamani, R.Z.Zhou, S. Agrawal, Pattern and kinetics of cytokine production following administration of phosphorothiate oligonucleotides in mice, Antisense Nucleic Acid Drug Dev. 7 (1997) 495-502.

[20] D. Hu, X. Su, R. Sun, G. Yang, H. Wang, J. Ren, et al., Human microsatellite DNA mimicking oligodeoxynucleotides down-regulate TLR9-dependent and -independent activation of human immune cells, Mol. Immunol. 46 (2009) 1387-1396.

[21] A. Trieu, T.L. Roberts, J.A. Dunn, M.J. Sweet, K.J. Stacey, DNA motifs suppressing TLR9 responses, Crit. Rev. Immunol. 26 (2006) 527-544.

[22] P. Lenert, Nucleic acid sensing receptors in systemic lupus erythematosus: development of novel DNA- and/or RNA-like analogues for treating lupus, Clin. Exp. Immunol. 161 (2010) 208-222.

[23] H. Shirota, I. Gursel, M. Gursel, D.M. Klinman, Suppressive oligodeoxynucleotides protect mice from lethal endotoxic shock, J. Immunol. 174 (2005) 4579-4583.

[24] H. Shirota, M. Gursel, D.M. Klinman, Suppressive oligodeoxynucleotides inhibit Th1 differentiation by blocking IFNg and IL-12 mediated signaling, J. Immunol. 173 (2004) 5002-5007.

[25] T. Sato, T. Shimosato, W.G. Alvord, D.M. Klinman, Suppressive oligodeoxynucleotides inhibit silica-induced pulmonary inflammation, J. Immunol. 180 (2008) 7648-7654.

[26] J.J. Kaminski, S.A. Schattgen, T.C. Tzeng, C. Bode, D.M. Klinman, K.A. Fitzgerald, Synthetic oligodeoxynucleotides containing suppressive TTAGGG motifs inhibit AIM2 inflammasome activation, J. Immunol. 191 (2013) 3876-3883.

[27] C. Bode, J. Wang, D.M. Klinman, Suppressive oligodeoxynucleotides promote the generation of regulatory T cells by inhibiting STAT1 phosphorylation, Int. Immunopharmacol. 23 (2014) 516-522.

[28] H.S. Li, S.S. Watowich, Innate immune regulation by STAT-mediated transcriptional mechanisms, Immunol. Rev. 261 (2014) 84-101.

[29] M. Muramatsu, K. Kinoshita, S. Fagarasan, S. Yamada, Y. Shinkai, T. Honjo, Class switch recombination and hypermutation require activation-induced cytidine deaminase (AID) a potential RNA editing enzyme, Cell 102 (2000) 553-563.

[30] V.A. Rathinam, Z. Jiang, S.N. Waggoner, S. Sharma, L.E. Cole, L. Waggoner, et al., The AIM2 inflammasome is essential for host defense against cytosolic bacteria and DNA viruses, Nat. Immunol. 11 (2010) 395-402.

[31] V. Hornung, A. Ablasser, M. Charrel-Dennis, F. Bauernfeind, G. Horvath, D.R. Caffrey, et al., AIM2 recognizes cytosolic dsDNA and forms a caspase-1-activating inflammasome with ASC, Nature 458 (2009) 514-518.

[32] L. Unterholzner, S.E. Keating, M. Baran, K.A. Horan, S.B. Jensen, S. Sharma, et al., IFI16 is an innate immune sensor for intracellular DNA, Nat. Immunol. 11 (2010) 997-1004.

[33] D.M. Klinman, D. Tross, S. Klaschik, H. Shirota, T. Sato, Therapeutic applications and mechanisms underlying the activity of immunosuppressive oligonucleotides, Ann. N. Y. Acad. Sci. 1175 (2009) 80-88.

[34] L. Dong, S. Ito, K.J. Ishii, D.M. Klinman, Suppressive oligonucleotides protect against collagen-induced arthritis in mice, Arthritis Rheum. 50 (2004) $1686-1689$

[35] N. Hernandez-Santos, S.L. Gaffen, Th17 cells in immunity to Candida albicans, Cell Host Microbe 11 (2012) 425-435.

[36] C. Fujimoto, D.M. Klinman, G. Shi, H. Yin, B.P. Vistica, J.D. Lovaas, et al., A suppressive oligodeoxynucleotide inhibits ocular inflammation, Clin. Exp. Immunol. 156 (2009) 528-534.

[37] J.M. Mazurek, P.L. Schleiff, J.M. Wood, S.A. Hendricks, A. Weston, Notes from the field: update: silicosis mortality-United States, 1999-2013, MMWR Morb. Mortal Wkly. Rep. 64 (2015) 653-654

[38] H. Kawasaki, A mechanistic review of silica-induced inhalation toxicity, Inhal. Toxicol. 27 (2015) 363-377.

[39] A.L. Franks, J.E. Slansky, Multiple associations between a broad spectrum of autoimmune diseases: chronic inflammatory diseases and cancer, Anticancer Res. 32 (2012) 1119-1136.

[40] M.J. Barnes, F. Powrie, Regulatory T cells reinforce intestinal homeostasis, Immunity 31 (2009) 401-411.
[41] P.B. De, T.J. Walsh, J.P. Donnelly, D.A. Stevens, J.E. Edwards, T. Calandra, et al., Revised definitions of invasive fungal disease from the European organization for research and treatment of cancer/invasive fungal infections cooperative group and the National Institute of Allergy and Infectious Diseases Mycoses study group (eortc/msg) consensus group, Clin. Infect. Dis. 46 (2008) 1813-1821.

[42] J. Jawien, The role of an experimental model of atherosclerosis: apoE-knockout mice in developing new drugs against atherogenesis, Curr. Pharm. Biotechnol. 13 (2012) 2435-2439.

[43] X. Cheng, Y. Chen, J.J. Xie, R. Yao, X. Yu, M.Y. Liao, et al., Suppressive oligodeoxynucleotides inhibit atherosclerosis in ApoE(-/-) mice through modulation of Th1/Th2 balance, J. Mol. Cell. Cardiol. 45 (2008) 168-175.

[44] C. Iadecola, J. Anrather, The immunology of stroke: from mechanisms to translation, Nat. Med. 17 (2011) 796-808.

[45] N. Zhang, X. Zhang, X. Liu, H. Wang, J. Xue, J. Yu, et al., Chrysophanol inhibits NALP3 inflammasome activation and ameliorates cerebral ischemia/reperfusion in mice, Mediators Inflamm. (2014), 370530.

[46] N. Deroide, X. Li, D. Lerouet, V.E. Van, L. Baker, J. Harrison, et al., MFGE8 inhibits inflammasome-induced IL-1beta production and limits postischemic cerebral injury, J. Clin. Invest. 123 (2013) 1176-1181.

[47] J. Zhao, Y. Mou, J.D. Bernstock, D. Klimanis, S. Wang, M. Spatz, et al., Synthetic oligodeoxynucleotides containing multiple telemeric TTAGGG motifs suppress inflammasome activity in macrophages subjected to oxygen and glucose deprivation and reduce ischemic brain injury in stroke-prone spontaneously hypertensive rats, PLoS One 10 (2015) e0140772.

[48] L.M. Coussens, Z. Werb, Inflammation and cancer, Nature 420 (2002) $860-867$.

[49] E.L. Abel, J.M. Angel, K. Kiguchi, J. DiGiovanni, Multi-stage chemical carcinogenesis in mouse skin: fundamentals and applications, Nat. Protoc. 4 (2009) 1350-1362.

[50] H. Ikeuchi, T. Kinjo, D.M. Klinman, Effect of suppressive oligodeoxynucleotides on the development of inflammation-induced papillomas, Cancer Prev. Res. (Phila.) 4 (2011) 752-757.

[51] T. Brown, Silica exposure, smoking: silicosis and lung cancer-complex interactions, Occup. Med. (Lond.) 59 (2009) 89-95.

[52] C. Bode, T. Kinjo, W.G. Alvord, D.M. Klinman, Suppressive oligodeoxynucleotides reduce lung cancer susceptibility in mice with silicosis, Carcinogenesis 35 (2014) 1078-1083.

[53] R. Takahashi, T. Sato, D.M. Klinman, T. Shimosato, T. Kaneko, Y. Ishigatsubo, Suppressive oligodeoxynucleotides synergistically enhance antiproliferative effects of anticancer drugs in A549 human lung cancer cells, Int. J. Oncol. 42 (2013) 429-436.

[54] H. Yamada, K.J. Ishii, D.M. Klinman, Suppressive oligodeoxynucleotides inhibit CpG-induced inflammation of the mouse lung, Crit. Care Med. 32 (2004) 2045-2049.

[55] P. Utaisincharoen, W. Kespichayawattana, N. Anuntagool, P. Chaisuriya, S Pichyangkul, A.M. Krieg, et al., CpG ODN enhances uptake of bacteria by mouse macrophages, Clin. Exp. Immunol. 132 (2003) 70-75.

[56] R.A. Zeuner, K.J. Ishii, M.J. Lizak, I. Gursel, H. Yamada, D.M. Klinman, et al., Reduction of GpG-induced arthritis by suppressive oligodeoxynucleotides, Arthritis Rheum. 46 (2002) 2219-2224.

[57] R.A. Zeuner, D. Verthelyi, M. Gursel, K.J. Ishii, D.M. Klinman, Influence of stimulatory and suppressive DNA motifs on host susceptibility to inflammatory arthritis, Arthritis Rheum. 48 (2003) 1701-1707.

[58] L.L. Stunz, P. Lenert, D. Peckham, A.K. Yi, S. Haxhinasto, M. Chang, et al., Inhibitory oligonucleotides specifically block effects of stimulatory CpG oligonucleotides in B cells, Eur. J. Immunol. 32 (2002) 1212-1222.

[59] A.M. Avalos, H.L. Ploegh, Competition by inhibitory oligonucleotides prevents binding of CpG to C-terminal TLR9, Eur. J. Immunol. 41 (2011) $2820-2827$.

[60] P. Lenert, W. Rasmussen, R.F. Ashman, Z.K. Ballas, Structural characterization of the inhibitory DNA motif for the type A (D)-CpG-induced cytokine secretion and NK-cell lytic activity in mouse spleen cells, DNA Cell Biol. 22 (2003) 621-631.

[61] P. Lenert, A.J. Goeken, R.F. Ashman, Extended sequence preferences for oligodeoxyribonucleotide activity, Immunology 117 (2006) 474-481.

[62] R.F. Ashman, J.A. Goeken, J. Drahos, P. Lenert, Sequence requirements for oligodeoxyribonucleotide inhibitory activity, Int. Immunol. 17 (2005) 411-420.

[63] E. Latz, A. Verma, A. Visintin, M. Gong, C.M. Sirois, D.C. Klein, et al., Ligand-induced conformational changes allosterically activate toll-like receptor 9, Nat. Immunol. 8 (2007) 772-779.

[64] P. Lenert, L. Stunz, A.K. Yi, A.M. Krieg, R.F. Ashman, CpG stimulation of primary mouse B cells is blocked by inhibitory oligodeoxyribonucleotides at a site proximal to NF-kappaB activation, Antisense Nucleic Acid Drug Dev. 11 (2001) 247-256.

[65] P. Lenert, A.K. Yi, A.M. Krieg, L.L. Stunz, R.F. Ashman, Inhibitory oligonucleotides block the induction of AP-1 transcription factor by stimulatory CpG oligonucleotides in B cells, Antisense Nucleic Acid Drug Dev. 13 (2003) 143-150.

[66] P. Lenert, K. Yasuda, L. Busconi, P. Nelson, C. Fleenor, R.S. Ratnabalasuriar, et al., DNA-like class R inhibitory oligonucleotides (INH-ODNs) preferentially block autoantigen-induced B-cell and dendritic cell activation in vitro and autoantibody production in lupus-prone MRL-Fas(lpr/lpr) mice in vivo, Arthritis Res. Ther. 11 (2009) R79. 
[67] F. Rommler, M. Jurk, E. Uhlmann, M. Hammel, A. Waldhuber, L. Pfeiffer, et al., Guanine modification of inhibitory oligonucleotides potentiates their suppressive function, J. Immunol. 191 (2013) 3240-3253.

[68] F. Rommler, M. Hammel, A. Waldhuber, T. Muller, M. Jurk, E. Uhlmann, et al., Guanine-modified inhibitory oligonucleotides efficiently impair, PLoS One 10 (2015) e0116703.

[69] P.I. Fields, R.V. Swanson, C.G. Haidaris, F. Heffron, Mutants of Salmonella typhimurium that cannot survive within the macrophage are avirulent, Proc. Natl. Acad. Sci. U. S. A. 83 (1986) 5189-5193.

[70] A. Trieu, N. Bokil, J.A. Dunn, T.L. Roberts, D. Xu, F.Y. Liew, et al., TLR9-independent effects of inhibitory oligonucleotides on macrophage responses to S. typhimurium, Immunol. Cell Biol. 87 (2009) 218-225.

[71] M. Peter, K. Bode, G.B. Lipford, F. Eberle, K. Heeg, A.H. Dalpke, Characterization of suppressive oligodeoxynucleotides that inhibit toll-like receptor-9-mediated activation of innate immunity, Immunology 123 (2008) 118-128.

[72] P.P. Ho, P. Fontoura, P.J. Ruiz, L. Steinman, H. Garren, An immunomodulatory GpG oligonucleotide for the treatment of autoimmunity via the innate and adaptive immune systems, J. Immunol. 171 (2003) 4920-4926.

[73] P.P. Ho, P. Fontoura, M. Platten, R.A. Sobel, J.J. DeVoss, L.Y. Lee, et al., A suppressive oligodeoxynucleotide enhances the efficacy of myelin cocktail/IL-4-tolerizing DNA vaccination and treats autoimmune disease, J. Immunol. 175 (2005) 6226-6234.

[74] K.L. Graham, L.Y. Lee, J.P. Higgins, L. Steinman, P.J. Utz, P.P. Ho, Treatment with a toll-like receptor inhibitory GpG oligonucleotide delays and attenuates lupus nephritis in NZB/W mice, Autoimmunity 43 (2010) $140-155$.

[75] M. Heikenwalder, M. Polymenidou, T. Junt, C. Sigurdson, H. Wagner, S. Akira, et al., Lymphoid follicle destruction and immunosuppression after repeated CpG oligodeoxynucleotide administration, Nat. Med. 10 (2004) 187-192.

[76] F. Fallarino, P. Puccetti, Toll-like receptor 9-mediated induction of the immunosuppressive pathway of tryptophan catabolism, Eur. J. Immunol. 36 (2006) 8-11.

[77] C. Volpi, F. Fallarino, R. Bianchi, C. Orabona, L.A. De, C. Vacca, et al., A $\mathrm{GpC}$-rich oligonucleotide acts on plasmacytoid dendritic cells to promote immune suppression, J. Immunol. 189 (2012) 2283-2289

[78] H. Yanai, A. Matsuda, J. An, R. Koshiba, J. Nishio, H. Negishi, et al., Conditional ablation of HMGB1 in mice reveals its protective function against endotoxemia and bacterial infection, Proc. Natl. Acad. Sci. U. S. A. 110 (2013) 20699-20704.

[79] O. Duramad, K.L. Fearon, B. Chang, J.H. Chan, J. Gregorio, R.L. Coffman, et al., Inhibitors of TLR-9 act on multiple cell subsets in mouse and man in vitro and prevent death in vivo from systemic inflammation, J. Immunol. 174 (2005) 5193-5200.

[80] R. Sun, L. Sun, M. Bao, Y. Zhang, L. Wang, X. Wu, et al., A human microsatellite DNA-mimicking oligodeoxynucleotide with CCT repeats negatively regulates TLR7/9-mediated innate immune responses via selected TLR pathways, Clin. Immunol. 134 (2010) 262-276.

[81] M. Fang, M. Wan, S. Guo, R. Sun, M. Yang, T. Zhao, et al., An oligodeoxynucleotide capable of lessening acute lung inflammatory injury in mice infected by influenza virus, Biochem. Biophys. Res. Commun. 415 (2011) 342-347.

[82] F.J. Barrat, T. Meeker, J. Gregorio, J.H. Chan, S. Uematsu, S. Akira, et al. Nucleic acids of mammalian origin can act as endogenous ligands for toll-like receptors and may promote systemic lupus erythematosus, J. Exp. Med. 202 (2005) 1131-1139.

[83] C. Guiducci, C. Tripodo, M. Gong, S. Sangaletti, M.P. Colombo, R.L. Coffman, et al., Autoimmune skin inflammation is dependent on plasmacytoid dendritic cell activation by nucleic acids via TLR7 and TLR9, J. Exp. Med. 207 (2010) 2931-2942.

[84] F.J. Barrat, T. Meeker, J.H. Chan, C. Guiducci, R.L. Coffman, Treatment of lupus-prone mice with a dual inhibitor of TLR7 and TLR9 leads to reduction of autoantibody production and amelioration of disease symptoms, Eur. J. Immunol. 37 (2007) 3582-3586

[85] R.D. Pawar, A. Ramanjaneyulu, O.P. Kulkarni, M. Lech, S. Segerer, H.J. Anders, Inhibition of toll-like receptor-7 (TLR-7) or TLR-7 plus TLR-9 attenuates glomerulonephritis and lung injury in experimental lupus, J. Am. Soc. Nephrol. 18 (2007) 1721-1731.

[86] P. Lenert, Inhibitory oligodeoxynucleotides-therapeutic promise for systemic autoimmune diseases? Clin. Exp. Immunol. 140 (2005) 1-10.
[87] P.S. Patole, D. Zecher, R.D. Pawar, H.J. Grone, D. Schlondorff, H.J. Anders, G-Rich DNA suppresses systemic lupus, J. Am. Soc. Nephrol. 16 (2005) 3273-3280.

[88] C. He, L. Zhou, R. Sun, T. Zhao, Y. Zhang, Y. Fu, et al., Effects of oligodeoxynucleotide with CCT repeats on chronic graft versus host disease induced experimental lupus nephritis in mice, Clin. Immunol. 140 (2011) 300-306.

[89] Y.Z. Wang, Q.H. Liang, H. Ramkalawan, W. Zhang, W.B. Zhou, B. Xiao, et al., Inactivation of TLR9 by a suppressive oligodeoxynucleotides can ameliorate the clinical signs of EAN, Immunol. Invest. 41 (2012) 171-182.

[90] Y.S. Zhang, X.L. Wu, Y. Wang, R. Sun, Y.L. Yu, L.Y. Wang, Structure-activity relationship of a guanine-free oligodeoxynucleotide as immunopotent inhibitor, Int. Immunopharmacol. 13 (2012) 446-453.

[91] D.A. Schwartz, T.J. Quinn, P.S. Thorne, S. Sayeed, Y. Ae, A.M. Krieg, CpG motifs in bacterial DNA cause inflammation in the lower respiratory tract, J. Clin. Invest. 100 (1997) 68-73.

[92] P. Markowski, O. Boehm, L. Goelz, A.L. Haesner, H. Ehrentraut, K. Bauerfeld, et al., Pre-conditioning with synthetic CpG-oligonucleotides attenuates myocardial ischemia/reperfusion injury via IL-10 up-regulation, Basic Res. Cardiol. 108 (2013) 376

[93] O. Boehm, P. Markowski, G.M. van der, V. Gielen, A. Kokalova, C. Brill, et al. In vivo TLR9 inhibition attenuates CpG-induced myocardial dysfunction, Mediators Inflamm. 2013 (2013) 217297.

[94] Y. Ito, S. Shigemori, T. Sato, T. Shimazu, K. Hatano, H. Otani, et al., Class I/II hybrid inhibitory oligodeoxynucleotide exerts Th1 and Th2 double immunosuppression, FEBS Open Bio 3 (2013) 41-45.

[95] H. Han, L.H. Hurley, G-Quadruplex DNA: a potential target for anti-cancer drug design, Trends Pharmacol. Sci. 21 (2000) 136-142.

[96] A.I. Murchie, D.M. Lilley, Retinoblastoma susceptibility genes contain 5 sequences with a high propensity to form guanine-tetrad structures, Nucleic Acids Res. 20 (1992) 49-53.

[97] R.F. Ashman, J.A. Goeken, P.S. Lenert, Aggregation and secondary loop structure of oligonucleotides do not determine their ability to inhibit TLR9, Int. Immunopharmacol. 11 (2011) 1032-1037.

[98] N. Jing, Y. Li, X. Xu, W. Sha, P. Li, L. Feng, et al., Targeting Stat3 with G-quartet oligodeoxynucleotides in human cancer cells, DNA Cell Biol. 22 (2003) 685-696.

[99] C.A. Stein, Y.C. Cheng, Antisense oligonucleotides as therapeutic agents-is the bullet really magical? Science 261 (1993) 1004-1012.

[100] K.J. Stacey, G.R. Young, F. Clark, D.P. Sester, T.L. Roberts, S. Naik, et al., The molecular basis for the lack of immunostimulatory activity of vertebrate DNA, J. Immunol. 170 (2003) 3614-3620.

[101] M. Jurk, A. Kritzler, B. Schulte, S. Tluk, C. Schetter, A.M. Krieg, et al., Modulating responsiveness of human TLR7 and 8 to small molecule ligands with T-rich phosphorothiate oligodeoxynucleotides, Eur. J. Immunol. 36 (2006) 1815-1826.

[102] D.M. Klinman, I. Gursel, S. Klaschik, L. Dong, D. Currie, H. Shirota Therapeutic potential of oligonucleotides expressing immunosuppressive TTAGGG motifs, Ann. N. Y. Acad. Sci. 1058 (2005) 87-95.

[103] R.F. Ashman, J.A. Goeken, E. Latz, P. Lenert, Optimal oligonucleotide sequences for TLR9 inhibitory activity in human cells: lack of correlation with TLR9 binding, Int. Immunol. 23 (2011) 203-214.

[104] K.L. Graham, L.Y. Lee, J.P. Higgins, L. Steinman, P.J. Utz, P.P. Ho, Treatment with a toll-like receptor inhibitory GpG oligonucleotide delays and attenuates lupus nephritis in NZB/W mice, Autoimmunity 43 (2010) $140-155$.

[105] P.P. Ho, P. Fontoura, M. Platten, R.A. Sobel, J.J. DeVoss, L.Y. Lee, et al., A suppressive oligodeoxynucleotide enhances the efficacy of myelin cocktail/IL-4-tolerizing DNA vaccination and treats autoimmune disease, J. Immunol. 175 (2005) 6226-6234.

[106] M.K. Wloch, S. Pasquini, H.C. Ertl, D.S. Pisetsky, The influence of DNA sequence on the immunostimulatory properties of plasmid DNA vectors Hum. Gene Ther. 9 (1998) 1439-1447.

[107] M.D. Halpern, D.S. Pisetsky, In vitro inhibition of murine IFN gamma production by phosphorothioate deoxyguanosine oligomers, Immunopharmacology 29 (1995) 47-52.

[108] P.S. Patole, H.J. Grone, S. Segerer, R. Ciubar, E. Belemezova, A. Henger, et al., Viral double-stranded RNA aggravates lupus nephritis through toll-like receptor 3 on glomerular mesangial cells and antigen-presenting cells, J. Am. Soc. Nephrol. 16 (2005) 1326-1338. 